\title{
Análisis con elemento finito y remalleo fractal en geotecnia
}

\section{Finite Element Analysis and Fractal Remeshing in Geotechnics}

\author{
Magaña del Toro R. \\ Instituto de Ingeniería \\ Universidad Nacional Autónoma de México \\ E-mail:rmat@pumas.iingen.unam.mx \\ Hermosillo-Arteaga A.R. \\ Instituto de Ingeniería \\ Universidad Nacional Autónoma de México \\ E-mail:AHermosilloA@iingen.unam.mx
}

\author{
Romo-Organista M.P. \\ Instituto de Ingeniería \\ Universidad Nacional Autónoma de México \\ E-mail:mromo@pumas.iingen.unam.mx \\ Carrera-Bolaños J. \\ Facultad de Ingeniería \\ Universidad Nacional Autónoma de México \\ E-mail:jorgec00@yahoo.com
}

Información del artículo: recibido: enero de 2009, aceptado: octubre de 2009

\section{Resumen}

La finalidad de este artículo es presentar de manera general las bases matemáticas de la geometría fractal y cómo este punto de vista geométrico se aplica a problemas que se presentan en el análisis de obras civiles complejas mediante el método del elemento finito, en el cual es importante la definición del tamaño de los elementos que componen la malla, de manera tal que la discretización del medio continuo sea representativa y consecuentemente los resultados obtenidos sean confiables. Para mostrar la aplicabilidad de esta técnica, se presenta un algoritmo fractal (desarrollado por los autores), mediante el cual se puede hacer remalleo automático de mallas bidimensionales de elemento finito, con lo cual se logran refinamientos locales eficientes de los problemas bajo análisis. Los resultados se comparan con soluciones analíticas mostrando que éstas se reproducen con excelente aproximación.

\footnotetext{
Abstract

The purpose of this article is to show in a general way the mathematical basis of the fractal geometry and how this geometrical point of view is applied to problems which appear in the finite element analysis of complex civil engineering structures, where the definition of the size of the elements that compose the mesh is important, such that the discretization of the continuum medium be representative and consequently the obtained results be more reliable. To show the applicability of this technology, a fractal algorithm (developed by the authors) is presented, by means of which it is possible to do automatic efficient local remeshing of finite element meshes for twodimensional stress analyses. The results are compared with analytical solutions which are reproduced with excellent approximation.
}

\section{Descriptores}

- elemento finito

- métodos numéricos

- geometría fractal

- mecánica del medio continuo

- remalleo automático

- problemas geotécnicos

- análisis de esfuerzos

\section{Keywords}

- finite element

- numerical methods

- fractal geometry

- continuum mechanics

- automatic remeshing

- geotechnical problems

- stress analysis 


\section{Introducción}

\section{Generalidades}

Objetivo. En este artículo se destaca la necesidad de vincular a los matemáticos modernos con los ingenieros civiles y en particular con los geotécnicos, logrando con ello concretizar el trabajo de los primeros y que los segundos puedan matematizar sus problemas técnicos.

Con el propósito de utilizar sus conceptos básicos para comprender los fundamentos matemáticos de la geometría fractal y sus elementos (fractales), en el apéndice de este artículo se comentan diferentes ramas de las matemáticas modernas (vinculadas con los fractales) como son: teoría de conjuntos, topología, teoría de la medida e integral de Lebesgue, etcétera.

Antecedentes y entorno. Con relación a este aspecto cabe mencionar que actualmente existe un vínculo muy escaso entre los matemáticos que realizan un trabajo típico de las matemáticas puras y los ingenieros que resuelven sus problemas casi de una manera intuitiva (aún hoy en día), con formulaciones o con modelos generalmente burdos.

Alcance. Por ser este un trabajo introductorio, únicamente se presentan algunas de las ramas de las matemáticas modernas de mayor impacto en la geometría fractal, destacando de cada una de ellas, aspectos que sean de interés para los ingenieros.

Finalmente, se presenta a manera de ejemplo un trabajo desarrollado por los autores para el remalleo automático de mallas de elemento finito basado en un algoritmo fractal y se aplica a problemas de ingeniería como son los túneles y cimentaciones.

\section{Reflexión sobre las matemáticas modernas}

La Matemática Moderna. Uno de los más trascendentales acontecimientos en la historia de la matemática es el planteamiento de la teoría de conjuntos, porque ha permitido alcanzar los niveles de generalización y abstracción que hoy predomina en la matemática moderna. El descubrimiento de las geometrías no euclidianas también contribuyó en la reestructuración de todo el edificio matemático. En la matemática clásica, sus diferentes ramas parecían desvinculadas. La matemática moderna se ha organizado de manera que las herramientas están agrupadas y ordenadas por estructuras matemáticas (Solís, 2004).

Matematización y concretización. La matematización es el proceso de construcción de un modelo matemático para casos prácticos. Un modelo matemático se define como la organización sistemática de un conjunto de conceptos matemáticos, basados en ciertos algoritmos, para dar solución a algún problema de la realidad concreta. La concretización es el proceso inverso a la matematización, es decir, es el proceso de transferir un modelo matemático a la realidad.

Tanto la matematización como la concretización deben ir desarrollándose y comprobándose mutuamente en un proceso dialéctico continuo y cada vez cualitativamente superior.

Se comienza la construcción de un modelo matemático cuando conocemos a profundidad la naturaleza (realidad concreta) y percibimos las relaciones entre sus partes.

Ideas sobre conceptos matemáticos básicos para fractales.

En este inciso se comentan brevemente las raíces matemáticas de los fractales. Puesto que los fractales son conjuntos compactos en los que se emplean álgebras sigma de tipo Borel, se deben tener claros algunos conceptos de conjuntos (vea el apéndice), asimismo la definición de compacto pertenece a la topología, también el comentario acerca de álgebras sigma, nos lleva al campo de las estructuras algebraicas (estos conceptos matemáticos subsecuentes se abordan brevemente en el apéndice. Por otra parte, una característica importante de los fractales es el empleo de sistemas iterativos de ecuaciones, los cuales implican transformaciones topológicas y conceptos de geometría algebraica y una consideración sobre el campo de soluciones de sistemas dinámicos no lineales (problemas caóticos). Finalmente, el concepto de dimensión fractal es algo que procede de la medida de Hausdorff, que en cierta forma es una generalización de la integral de Lebesgue, que a su vez es aplicable a conjuntos más generales a los que se aplica la integral de Riemann. Todo esto cae dentro del campo de la teoría de la medida, la cual tiene como caso particular la teoría de probabilidades. Por todo esto, es recomendable que se tengan en ingeniería al menos unas nociones básicas de todas estas raíces de los fractales (vea el apéndice).

\section{Matemáticas fractales}

\section{Introducción}

Durante muchos años los sistemas y modelos lineales han sido utilizados sistemáticamente para describir y modelar la dinámica de muchos sistemas físicos, quími- 
cos, económicos, etcétera. Sin embargo, en los últimos años se ha comprobado que los sistemas no lineales pueden presentar dinámicas muy complejas que no pueden aproximarse mediante modelos lineales o cuasilineales. Quizás el más claro ejemplo de este fenómeno lo constituyen los conjuntos de Mandelbrot y de Julia. Estos conjuntos son la representación gráfica de los infinitos comportamientos que se obtienen al iterar una ecuación no lineal (una ecuación cuadrática). Entre la gran variedad de comportamientos posibles de un sistema no lineal, el conocido por caos determinista destaca por su complejidad (Gutiérrez, 1998). Los sistemas caóticos son sensibles a pequeñas perturbaciones externas y, por tanto, se comportan de forma impredecible, a pesar de estar definidos por ecuaciones deterministas. La geometría fractal y la teoría de los sistemas dinámicos están íntimamente ligadas, ya que la región del espacio hacia la que tiende asintóticamente una órbita caótica tiene estructura fractal (atractores extraños). Los objetos fractales tienen propiedades muy particulares, como la autosemejanza y la apariencia irregular, que permiten caracterizarlos con base en medidas cuantitativas relativas a su grado de irregularidad. La más popular de estas medidas cuantitativas es la dimensión fractal, una extensión de la dimensión euclidiana para objetos autosemejantes.

\section{Geometría fractal}

La geometría tradicional (euclidiana) se encarga de las propiedades y de las mediciones de objetos tales como puntos, líneas, planos y volúmenes. A diferencia de estos objetos geométricos clásicos, que poseen propiedades de continuidad y diferenciabilidad, existen otros objetos geométricos irregulares que presentan una misma estructura a cualquier escala y tienen un número infinito de singularidades (puntos no diferenciables). Ejemplos de estos objetos son las formas encontradas en la naturaleza, como montañas, franjas costeras, sistemas hidrográficos, nubes, hojas, árboles, y un sinnúmero de otros objetos que no es fácil describir mediante la geometría tradicional. La geometría fractal provee una descripción matemática de estas formas irregulares que se denominan fractales (Barnsley, 1990). Una de las principales características de los fractales es la invariancia a los cambios de escala; es decir, un objeto fractal posee estructura a cualquier escala formada por copias de sí mismo a menor escala.

El desarrollo de la geometría fractal ha permitido obtener parámetros cuantitativos para definir el "grado de irregularidad" de un determinado objeto. Esto es un criterio basado en teoría de la medida (vea el apéndice), en la que se cuantifican características de un conjunto cualesquiera mediante una cantidad escalar.

Características de los fractales. Las características que definen un fractal son las siguientes:

- Autosimilitud. A diferentes escalas, un fractal conserva la misma apariencia, siempre existe una clara similitud entre partes muy distantes de una misma figura fractal.

- Infinito detalle. Relacionada con la característica anterior, al ampliar un fractal, tanto más detalle revela éste, sin que se tenga un límite en el que se aprecien curvas suavizadas.

- Dimensión no entera. Al contrario de la geometría clásica, en la que las figuras tienen 1, 2 ó 3 dimensiones, un fractal puede desarrollarse en una dimensión no entera, por ejemplo la curva de Koch, que lo hace en la dimensión 1.26; esto es, ocupa parte del plano pero no llega a tener la entidad de figura bidimensional. Esta dimensión se deduce a partir de la medida de Hausdorff.

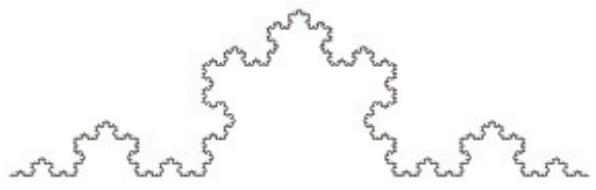

Figura 1. Curva de Koch

- Las fórmulas o algoritmos que los definen son relativamente sencillos y operan con un conjunto muy reducido de datos.

- Su algoritmia es definida por una característica clave: la iteración. La aparición en la ingeniería de las computadoras es lo que permite experimentar y descubrir nuevos conjuntos y sin ellas, probablemente Mandelbrot no hubiese llegado tan lejos.

Modelos fractales. En la actualidad existen numerosos modelos matemáticos que permiten definir objetos fractales asociados con problemas particulares. Por ejemplo, se puede construir una curva de interpolación fractal asociada a un conjunto de puntos; también son populares los paisajes fractales creados por computadoras.

Uno de los modelos matemáticos más popular para crear objetos fractales es el conocido como sistema de funciones iteradas (IFS). En este caso, el fractal está definido como el único subconjunto invariante a la unión de un conjunto finito de transformaciones lineales que forman el IFS (por tanto, el fractal se puede descomponer en un conjunto finito de copias afines de sí mismo). 
Estos modelos se analizan con detalle en (Falconer, 1993). Como ejemplo de aplicación de la geometría fractal se tiene la compresión de imágenes (Gutiérrez, 1998). Aún cuando una imagen no posea aparentemente la propiedad de autosemejanza, es posible que algunas partes de ella sean semejantes a otras. Por tanto, si encontráramos las transformaciones lineales necesarias para que distintos subconjuntos de la imagen se transformen en otros, podríamos reemplazar la imagen por las transformaciones que la definen.

Ejemplos de iteración (los conjuntos de Julia y Mandelbrot). Antes se mencionó que la dinámica iterativa de ecuaciones no lineales puede dar lugar a una gran variedad de comportamientos. La teoría de sistemas dinámicos estudia aquellos modelos que evolucionan en el tiempo y que pueden ser descritos bien mediante funciones o mapas (sistemas discretos), o bien mediante ecuaciones diferenciales (sistemas continuos). Por ejemplo, los conjuntos de Mandelbrot y Julia resultan del sistema dinámico discreto dado por el mapeo $z=c^{2}+a$.

\section{Dimensión fractal}

La propiedad de auto-similitud o escalamiento es uno de los conceptos centrales de geometría fractal. Este concepto se encuentra muy unido al de dimensión fractal, como se ve en la figura 2 (Muñoz, 2007).

Como ejemplo del cálculo de la dimensión fractal tenemos lo siguiente:

Si tomamos un segmento de longitud 1, y lo partimos en segmentos de longitud L obtendremos $\mathrm{N}$ partes, de manera que $N^{*} L^{1}=1$, cualquiera que sea $L$ (figura 2a).

Si el objeto inicial es un cuadrado de superficie 1 y lo comparamos con unidades cuadradas, cuyo lado tenga de longitud $L$, el número de unidades que es necesario para recubrirlo $N$, se cumple $N^{*} L^{2}=1$ (figura $2 b$ ).

Si por último, el objeto que tomamos es tridimensional, por ejemplo un cubo de volumen 1 , y lo medimos con relación a unidades que sean cubos de arista $L$, entonces se cumple que $N^{*} L^{3}=1$ (figura 2c).

De todo esto podemos generalizar que la dimensión fractal de un objeto geométrico es $D$ si:

$N^{*} L^{D}=1$

donde $N$ es el número de objetos elementales, o de unidades, de tamaño $L$ que recubren o que completan el objeto. Despejando $D$ :

$D=\frac{\log (N)}{\log (1 / L)}$
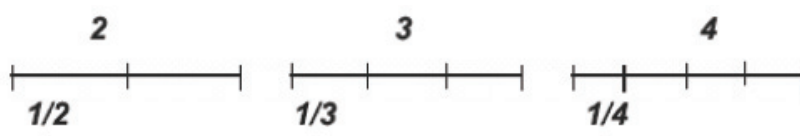

(a)

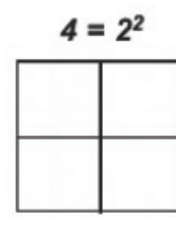

$1 / 2$

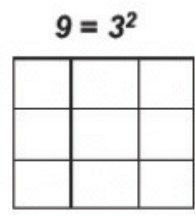

$1 / 3$

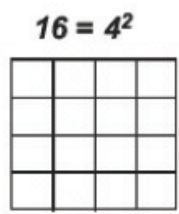

$1 / 4$

(b)
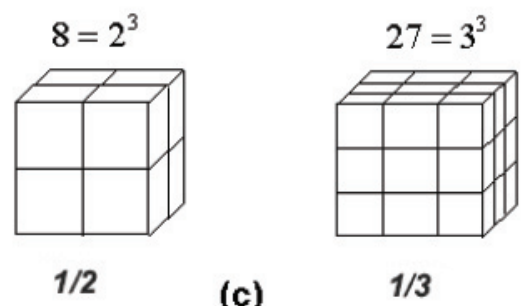

(c)

Figura 2. Dimensiones 1 a) , 2 b) y 3 c)

Así por ejemplo, analizando la curva de Koch (figura 3) tenemos:

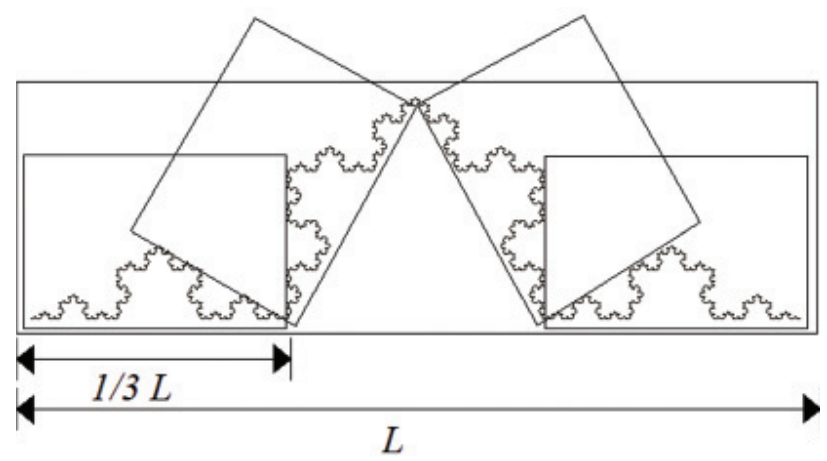

Figura 3. Cálculo de la $D$ de la curva de Koch

Reduciendo la escala de la curva a $1 / 3$ encontramos que se descompone en 4 partes, y utilizando la ecuación 2, se tiene:

$D=\frac{\log (4)}{\log (3)}=1.2618$

A medida que $D$ aumenta de uno a dos, las curvas resultantes comienzan a pasar de "parecidas a líneas" a "llenar planos". El mismo fenómeno ocurre con las figuras con dimensión entre 2 y 3, cada vez comienzan a llenar más el espacio. 
Sistemas de funciones iteradas

Generalidades. Es claro ver en la naturaleza que muchos fenómenos tienen autosimilitud. Ejemplos hay varios, como las hojas de helechos, donde una de ellas está compuesta por muchas hojas pequeñas. Estas a su vez, compuestas de hojas más pequeñas aún; otro ejemplo son las nubes.

Por tanto, la característica de autosimilitud de estos fenómenos debería ser representable a través de modelación fractal (Guzmán, 1993 y Falconer, 2003).

Uno de los métodos más usados para modelar estos fenómenos son las IFS o sistemas de funciones iteradas.

Básicamente las IFS se pueden entender como un conjunto de $\mathrm{N}$ ecuaciones de transformación en un espacio real

$\operatorname{IFS}=\left\{w_{n^{\prime}} p_{n}: n=1,2, \ldots, N\right\}$

donde las $w_{n}$ son cada una de las transformaciones y las $p_{n}$ son probabilidades que definiremos más adelante. Lo que caracteriza a las transformaciones es que deben ser contractantes, es decir, si tengo dos puntos separados por una distancia $d$, al aplicarles cualquiera de estas transformaciones se obtiene un par de puntos separados por una distancia $d^{\prime}<d$.

Las probabilidades $p_{n}$ en tanto, definen, dado un punto cualquiera, que sobre él se aplique alguna de las $N$ transformaciones. La forma de usar las IFS es tomando un punto cualquiera en el espacio y aplicarle aleatoriamente (según las probabilidades $p_{n}$ ) alguna de las $N$ transformaciones para obtener el siguiente punto. Al continuar este proceso un número infinito de veces se obtiene una sucesión de puntos que definen la IFS. Un ejemplo clarificador es la obtención del triángulo de Sierpinski a través de este método. La forma de hacerlo es:

1. Se definen tres puntos vértice cualesquiera y los llamamos $A, B$ y $C$ (figura 4 ).

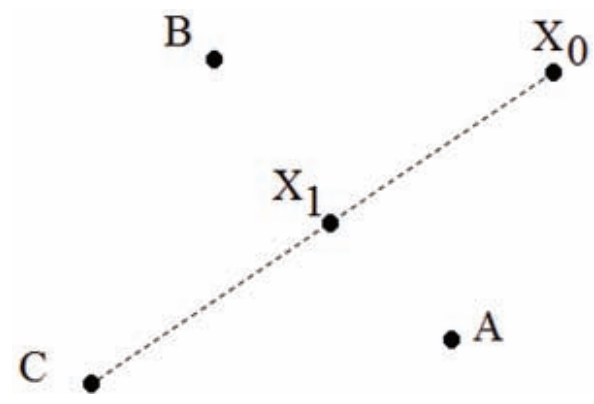

Figura 4. Primer paso de la IFS para obtener el triángulo de Sierpinski
2. Se define un punto $X_{0}$ cualquiera, como se ve en la figura 4.

3. Con probabilidad $1 / 3$ elegimos cualquiera de los tres puntos vértice, y tomamos como nuevo elemento de la sucesión al punto ubicado a la mitad del camino entre $X_{0}$ y el vértice escogido. En la figura se puede ver cuál sería $X_{1}$ en el caso de que el vértice utilizado hubiese sido $C$.

4. $X_{2}$ se obtiene con el mismo proceso sobre $X_{1}$.

5. Obteniendo muchos puntos de la sucesión y borrando estratégicamente algunos de los puntos iniciales, se puede obtener el triángulo de Sierpinski, como se ve en la figura 5 .

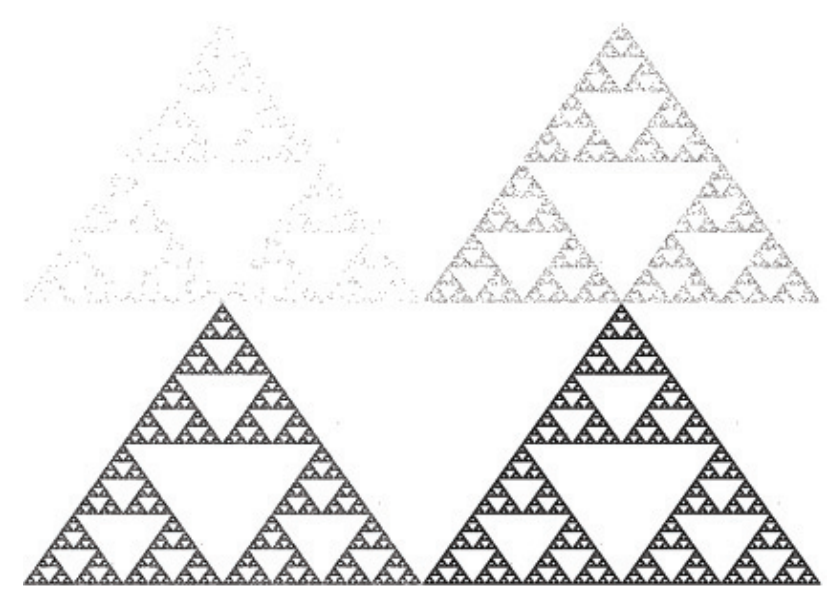

Figura 5. Triángulo de Sierpinski obtenido vía IFS

Fractales aleatorios. Los fractales pueden ser básicamente de dos formas. Están los que se componen de muchas copias escaladas y rotadas de sí mismas como el copo de nieve de Von Koch o el triángulo de Sierpinski. Incluso el conjunto complejo de Julia cae en esta categoría puesto que su estructura está contenida idénticamente en sí misma. La generación gráfica de estas figuras requiere una regla básica que se repite una y otra vez, generalmente de forma recursiva. En los fractales que queremos construir se incluye un elemento de aleatoriedad, intentando así, simular fenómenos naturales. A estos fractales les llamaremos fractales aleatorios.

Dado que los fractales tienen detalles infinitos en todas las escalas, la representación completa de un fractal en un computador es imposible. El nivel deseado de resolución queda limitado por el número disponible de pixeles que tenemos para desplegar el gráfico, o la cantidad de tiempo para hacer cálculos por computador que uno quiere invertir. 


\section{Aproximación fractal de objetos}

Introducción. Si tenemos unas semejanzas contractivas (son transformaciones topológicas contractivas, como se mencionó antes, $f_{1}, f_{2}, \ldots, f_{m}$ es decir aplicaciones $f_{1}: \Re_{n} \rightarrow \Re_{n}$ (transformaciones en un espacio real $n$-dimensional), tales que:

$d\left(f_{1}(x), f_{1}(y)\right)=r d(x, y)$, con $0<r<1$,

y para un conjunto $B$ compacto (implica una propiedad del conjunto, en la cual todos sus elementos son manejables, es decir, acotados). Esto es, a todos los puntos del conjunto se les puede aplicar la transformación. Así, si tomamos el operador $S$ y lo aplicamos a todos los puntos del conjunto $B$, se tiene:

$$
S(B)=\bigcup_{i=1}^{m} f_{i}(B)
$$

siendo entonces $S$, la unión de una serie de transformaciones $f_{i^{\prime}}$ que pueden representar un sistema de funciones iteradas. Entonces si se realizan $n$ iteraciones se tendrá:

$B \rightarrow S(B) \rightarrow S(S(B))=S^{2}(B) \rightarrow S\left(S^{2}(B)\right) \rightarrow \ldots \rightarrow F$

donde en la simbología anterior las flechas indican la aplicación iterada de la transformación $S$. Luego si se hacen $n$ iteraciones se llega a:

$S(F)=S\left(\lim _{n \rightarrow \infty} S^{n}(B)\right)=\lim _{n \rightarrow \infty} S^{n+1}(B)=F$

Nótese que después de $n$ iteraciones se converge al conjunto fractal $F$.

Por ejemplo, si tomamos el conjunto $B$ como todos los puntos de un cuadrado y a las transformaciones del plano $w_{1}, w_{2} \mathrm{y} w_{3}$ (donde cada una de ellas es un operador matricial) se tendrá:

$$
\begin{aligned}
& w_{1}\left(\begin{array}{l}
x \\
y
\end{array}\right)=\left(\begin{array}{cc}
1 / 2 & 0 \\
0 & 1 / 2
\end{array}\right)\left(\begin{array}{l}
x \\
y
\end{array}\right) \\
& w_{2}\left(\begin{array}{l}
x \\
y
\end{array}\right)=\left(\begin{array}{cc}
1 / 2 & 0 \\
0 & 1 / 2
\end{array}\right)\left(\begin{array}{l}
x \\
y
\end{array}\right)+\left(\begin{array}{c}
1 / 2 \\
0
\end{array}\right) \\
& w_{3}\left(\begin{array}{l}
x \\
y
\end{array}\right)=\left(\begin{array}{cc}
1 / 2 & 0 \\
0 & 1 / 2
\end{array}\right)\left(\begin{array}{l}
x \\
y
\end{array}\right)+\left(\begin{array}{c}
1 / 4 \\
\sqrt{3} / 4
\end{array}\right)
\end{aligned}
$$

En la figura 6, el primer cuadrado a la izquierda, se transforma en los tres cuadrados más pequeños contiguos (cada uno es el resultado de cada una de las tres transformaciones $\left.w_{1}, w_{2} \mathrm{y} w_{3}\right)$. Así mismo, si a cada uno de estos tres cuadrados se les aplica de nuevo las mismas tres transformaciones, se generarán la serie de nueve cuadrados que aparecen a la derecha de dicha figura 6. Si el proceso se continúa repitiendo, es decir iterando, se tendrán los conjuntos resultantes que aparecen en las figuras 7 y 8 .
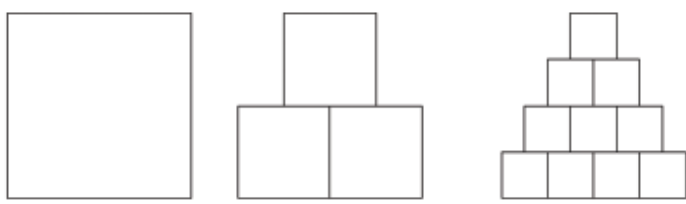

Figura 6.
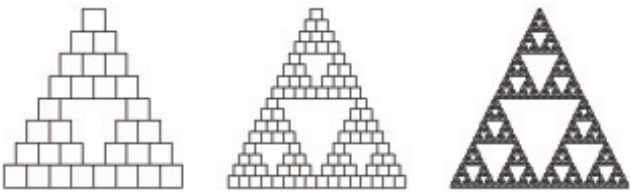

Figura 7.
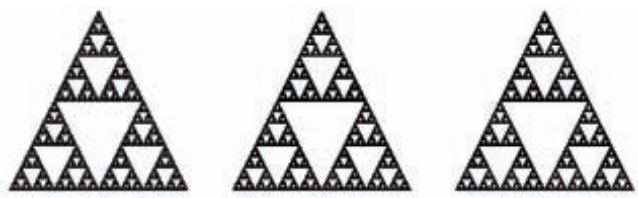

Figura 8.

De la misma manera se pueden generar otros objetos más complejos. Para ello debemos afinar nuestras herramientas definiendo algo más general que las semejanzas contractivas, (Barnsley, 1985). En la práctica, serán aplicaciones de la forma:

$f(x, y)=\left(\begin{array}{ll}a & b \\ c & d\end{array}\right)\left(\begin{array}{l}x \\ y\end{array}\right)+\left(\begin{array}{l}e \\ f\end{array}\right)$

Estas se llaman aplicaciones afines. Este es otro concepto topológico.

Atractores de sistemas de funciones iteradas. Si tomamos unas funciones contractivas:

$S=\left\{g_{1}, g_{2}, \ldots, g_{m}\right\}$

(un sistema de funciones iteradas o SFI), siempre existe un único conjunto $F$ tal que:

$F=\bigcup_{i=1}^{m} g_{i}(F)$

$F$ se llama atractor del sistema $S$.

$F$ es "autosemejante" según las transformaciones $S$. 
Métodos para determinar atractores. Para obtener el conjunto $F$ tal que:

$F=S(F)=\bigcup_{i=1}^{m} g_{i}(F)$

existen dos procedimientos: uno determinista y otro aleatorio.

Método determinista. Tomamos un conjunto compacto B y construimos

$B \rightarrow S(B) \rightarrow S(S(B))=S^{2}(B) \rightarrow S\left(S^{2}(B)\right) \rightarrow \ldots$

Tomando límites cuando $n$ tiende a infinito,

$F=\lim _{n \rightarrow \infty} S^{n}(B)=\lim _{n \rightarrow \infty} S^{n}\left(S^{n}(B)\right)=S\left(\lim _{n \rightarrow \infty} S^{n}(B)\right)=S(F)$

Es decir, partiendo de cualquier $B$ llegamos al atractor $F$.

Método aleatorio. Si $S=\left\{g_{1}, g_{2}, \ldots, g_{m}\right\}$, tomamos $x_{0}$ (cualquiera). Elegimos al azar

$x_{1} \in\left\{g_{1}\left(x_{0}\right) \cdot g_{2}\left(x_{0}\right), \ldots, g_{m}\left(x_{0}\right)\right\}$.

A continuación, elegimos al azar

$x_{2} \in\left\{g_{1}\left(x_{1}\right) \cdot g_{2}\left(x_{1}\right), \ldots, g_{m}\left(x_{1}\right)\right\}$,

$x_{3} \in\left\{g_{1}\left(x_{2}\right) \cdot g_{2}\left(x_{2}\right), \ldots, g_{m}\left(x_{2}\right)\right\} \ldots$,

construyendo una sucesión de puntos $\left(x_{n}\right)$ que cumple que: $\lim _{n \rightarrow \infty} x_{n}=x \in F$.

Repitiendo con otros (muchos) $x_{0}$, obtenemos una aproximación de $F$, es decir usando esta técnica se logra la aproximación de objetos mediante SFI.

Teorema del collage. Para modelar la naturaleza vía IFS es necesario encontrar algunas funciones contractantes que den la simetría necesaria a nuestro dibujo. Es claro que teniendo dos versiones de una misma imagen, donde la segunda es una versión escalada y rotada de la primera, se puede obtener la función contractante de esta transformación tomando algunos de los vértices (tantos como sean necesarios) para formar un sistema de ecuaciones donde las incógnitas sean las constantes de la transformada.

La generación de modelos nubosos es un ejemplo muy clarificador. Cada una de las pequeñas copias define una función contractiva que formará parte del conjunto de las IFS para la modelación del objeto.
Aproximación de una hoja (ejemplo). Construimos contracciones afines del tipo (4) y empleamos la siguiente tabla de datos:

\begin{tabular}{|c|c|c|c|c|c|}
\hline$a$ & $b$ & $C$ & $d$ & $e$ & $f$ \\
\hline 0.14 & 0.01 & 0 & 0.51 & -0.08 & -1.31 \\
\hline 0.43 & 0.52 & -0.45 & 0.50 & 1.49 & -0.75 \\
\hline 0.45 & -0.49 & 0.47 & 0.47 & -1.62 & -0.74 \\
\hline 0.49 & 0 & 0 & 0.51 & 0.02 & 1.62 \\
\hline
\end{tabular}

Partiendo inicialmente de la figura 9, al realizar las aplicaciones contractivas indicadas por la tabla anterior, se generan sucesivamente las figuras 10 y 11 . Este proceso se puede repetir las veces que se quiera.

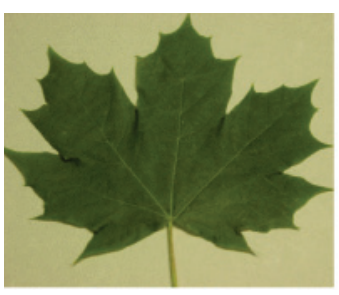

Figura 9.

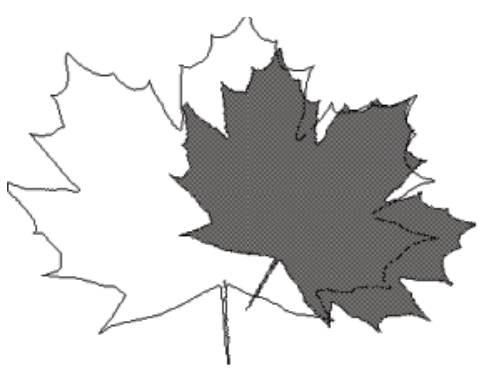

Figura 10.

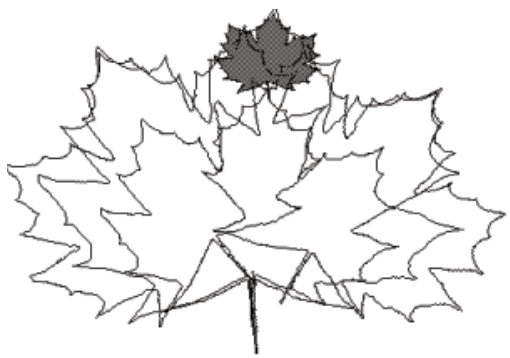

Figura 11.

Observe que, en las figuras 10 y 11, se ha marcado (mediante el contorno sombreado) una de las varias transformaciones realizadas a partir de la figura 9, la cual aparece contraída.

Esta misma situación se puede repetir con muchos objetos. 


\section{Remalleo fractal automático}

En esta sección se aplica un algoritmo de remalleo automático desarrollado en el Instituto de Ingeniería de la UNAM por los autores, para analizar el campo de deformaciones en túneles y cimentaciones superficiales. Con el remalleo automático se afina la malla de elementos finitos en la zona que se excede el criterio de falla; con ello se mejoran los resultados numéricos en áreas donde existen cambios bruscos en las propiedades de materiales adyacentes (por ejemplo interacción suelo-estructura, núcleo arcilloso y transiciones en presas, etcétera). Es interesante notar que en los casos analizados se ha podido constatar, que las regiones de posibles superficies de falla son parecidas a las que se observan en la realidad.

\section{Algoritmo de remalleo automático}

El remalleo se realiza automáticamente en las áreas en las que el criterio de falla adoptado sea excedido. Determinados los elementos que deben ser "fragmentados", se recalcula el estado de esfuerzos y se verifica nuevamente si éstos (en toda la malla) violan o no el criterio de falla. Este proceso es iterativo y se detendrá hasta que se satisfaga el criterio adoptado.

Recurriendo a la teoría de fractales, el algoritmo para la generación del triángulo de Sierpinsky ofrece una solución al problema de remalleo, debido a que los triángulos son adecuados para subdividir la malla sólo en algunos sitios, sin alterar su totalidad, como puede observarse (más adelante) en la zona dentro del círculo de la figura 13.

Los tres pasos básicos para la generación de mallas de elemento finito son (Magaña et al., 2001):

1. generación de nudos,

2. construcción de vínculos entre nudos para formar los elementos,

3. empleo de funciones de densidad para redistribuir adecuadamente los nudos y formar mallas de buen aspecto en la zona crítica.

La geometría fractal se aplica para la generación de nudos, posteriormente se emplean las técnicas comunes de elemento finito.

Procedimiento seguido. Se elaboró un algoritmo en lenguaje fortran para realizar la formación de las incidencias de los elementos generados en cada etapa de remalleo, así como el cálculo de las coordenadas de los nudos generados, con lo cual se logra tener los datos necesarios para iniciar los análisis de elemento finito después de cada refinamiento (Magaña et al., 2003). A partir de una malla inicial a base de triángulos (de preferencia equiláteros), se toma como conjunto iniciador un sistema de cuatro triángulos contiguos cualesquiera (de dicha malla), vea la figura 12 .

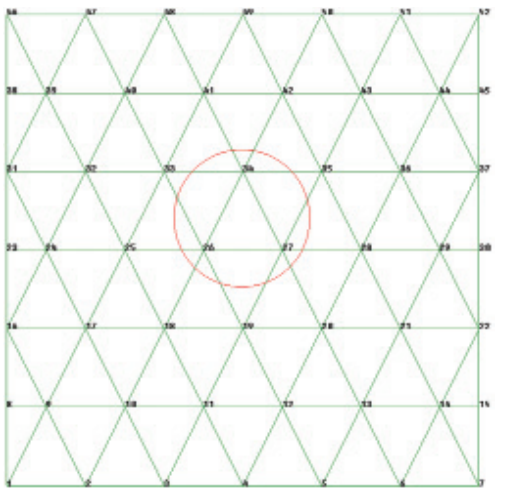

Figura 12. Malla de elemento finito inicial

El triángulo central se toma como elemento generador, el cual se fracciona en cuatro elementos aplicando el procedimiento de Sierpinsky, creando un nuevo triángulo central cuyos vértices son los puntos medios de los lados del triángulo central inicial. Por otra parte, se dividen a la mitad los otros tres triángulos -los que rodean al central inicial- (figuras 13 y 14). Con esto, el sistema original de cuatro triángulos, en dos iteraciones, se convierte en un conjunto de 10 y 16 triángulos, respectivamente.

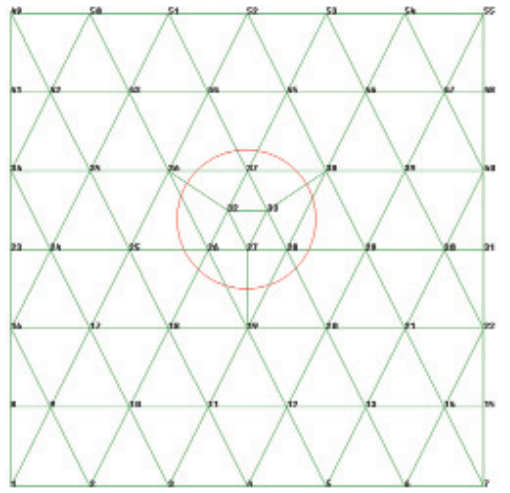

Figura 13. Malla de elemento finito refinada una vez

En cada iteración, los seis elementos adicionales a los cuatro internos, constituyen el cambio hecho al método de Sierpinsky, con lo que se evitan nudos inválidos en una malla de elemento finito. La característica fractal de este procedimiento es que se puede aplicar reiterada- 


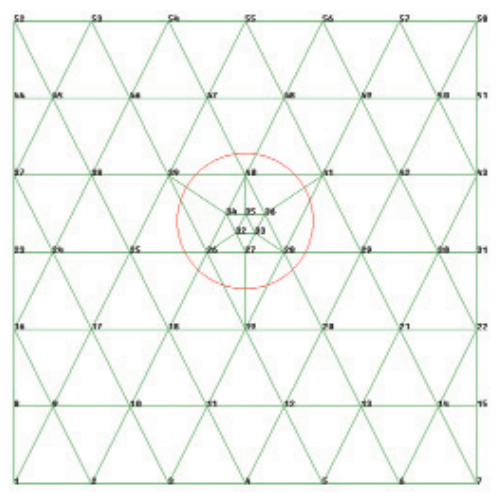

Figura 14. Malla de elemento finito refinada dos veces

mente a cada elemento de las mallas modificadas y en cualquier región, como se ejemplifica en la malla de la figura 13, dando como resultado la malla de la figura 14. En la figura 15 se presenta el diagrama de flujo del proceso de remalleo automático en un análisis con elemento finito.

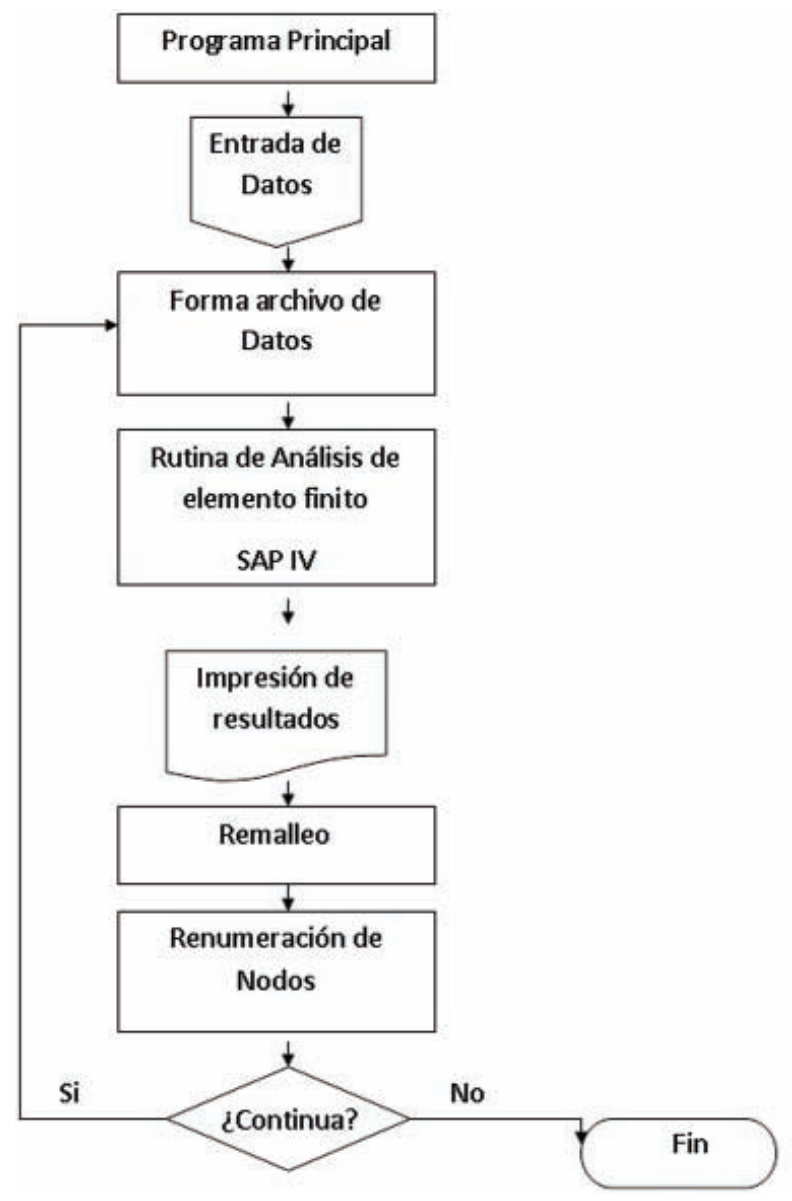

Figura 15. Diagrama de flujo del proceso de remalleo automático

\section{Evaluación del algoritmo}

Con objeto de comprobar la potencialidad y eficacia que ofrece el algoritmo de remalleo fractal, se analizó el caso (bidimensional) de un túnel en un espacio infinito y sometido a cargas vertical y horizontal como se indica en la figura 16. Los resultados obtenidos mediante el método del elemento finito (MEF) se comparan con la solución analítica elástico-lineal y elasto-plástica para este problema. De la misma forma, se presenta la solución con MEF para tres tamaños de malla: a) burda, b) intermedia y c) fina.

Solución analítica elástico-lineal para un túnel circular

Se considera un túnel de sección circular aislado, suficientemente largo (estado plano de deformaciones), sin revestimiento.

La solución elástica lineal fue propuesta por Kirsch y es un caso similar al de una placa infinita con un orificio circular en el centro (figura 16).

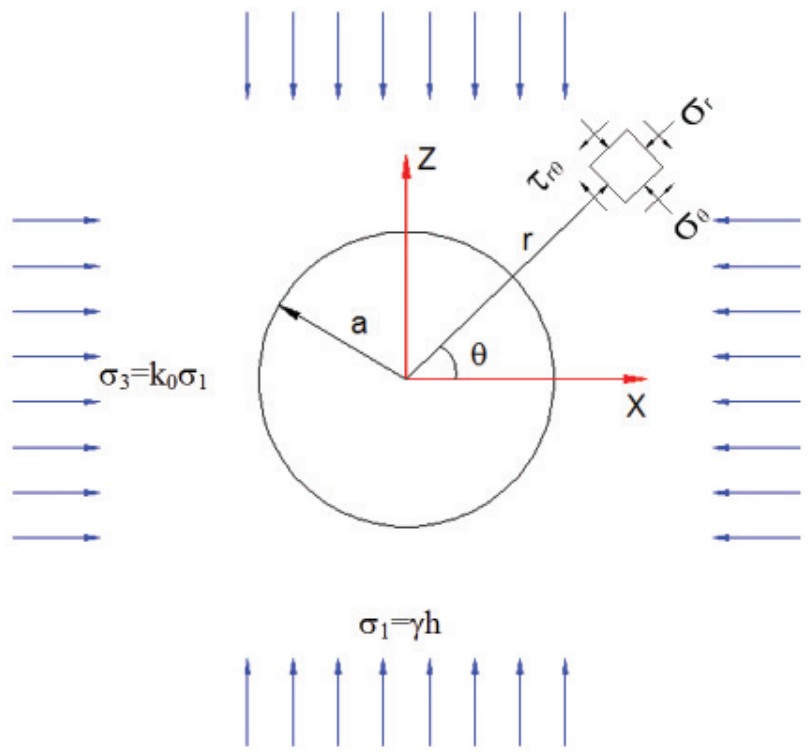

Figura 16. Análisis de un túnel en un medio infinito

Las expresiones para calcular los desplazamientos pueden consultarse en la referencia Obert y Duval (1967).

Análisis elástico-lineal por el método del elemento finito. Se analizó un túnel sin revestimiento en una masa de suelo mediante el método del elemento finito. Para ello se modeló el túnel con tres mallas de elementos finitos triangulares, variando el tamaño promedio 
de los elementos para cada malla (vea las figuras $17 \mathrm{y}$ 18); esto con el fin de mostrar que el tamaño de los elementos influye en la convergencia de los resultados obtenidos a la solución analítica.

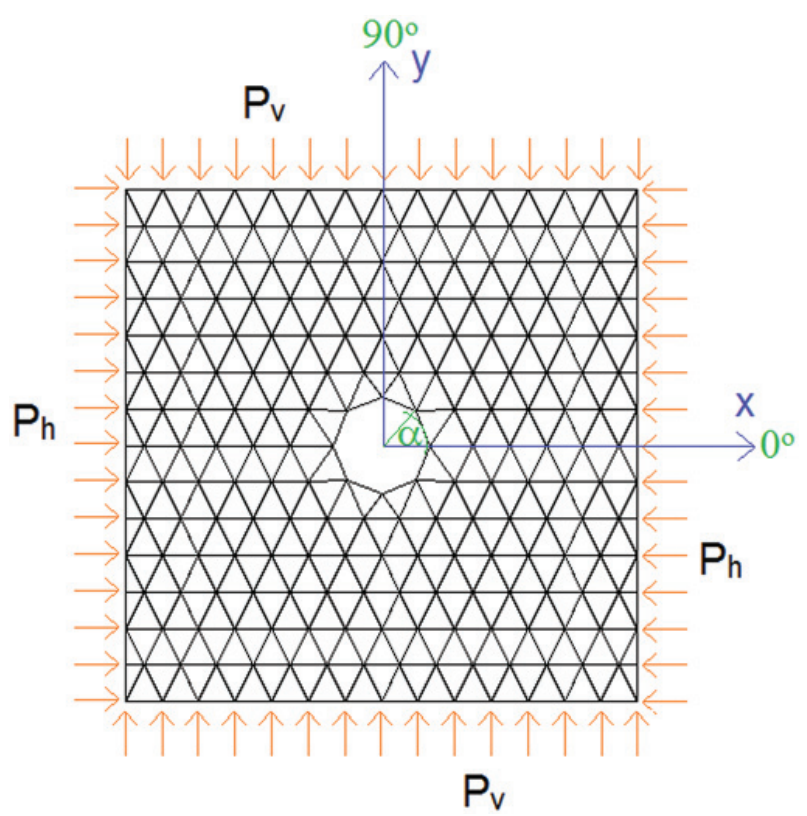

Figura 17. Malla burda. 396 elementos y 232 nudos

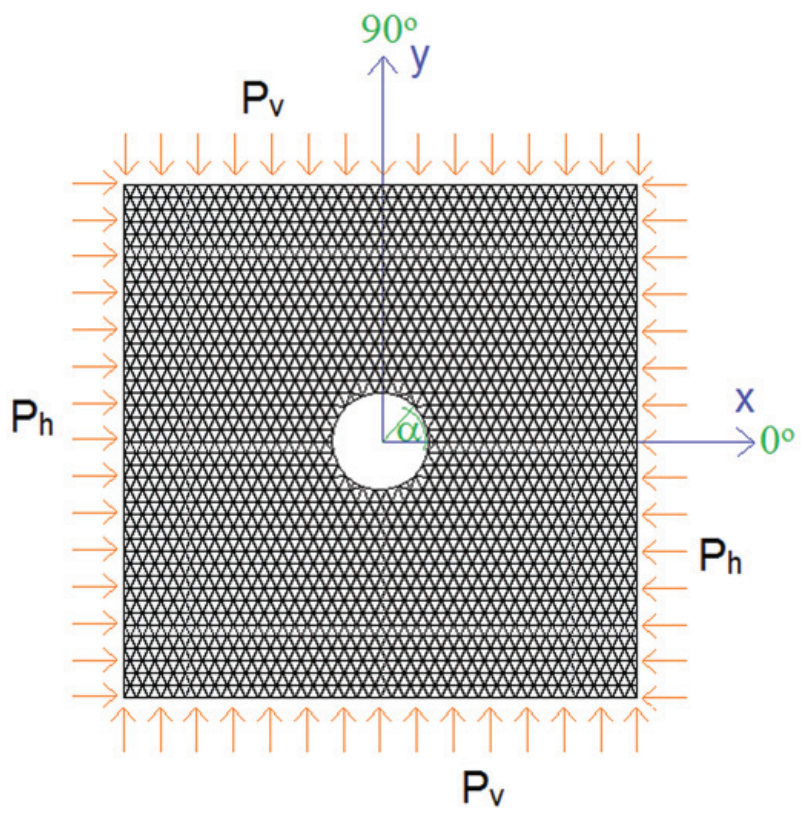

Figura 18. Malla fina de 3470 elementos y 1870 nudos

Posteriormente, se aplicó el algoritmo de remalleo fractal sobre la malla burda. En la figura 19 puede observar- se que el remalleo se concentró muy cerca del orificio. Los elementos generados por el remalleo tienen un tamaño mucho menor que los elementos iniciales, incluso menores a los de la malla fina. Sin embargo, el total de elementos y nudos es menos de la mitad de los que se tienen en la malla fina.

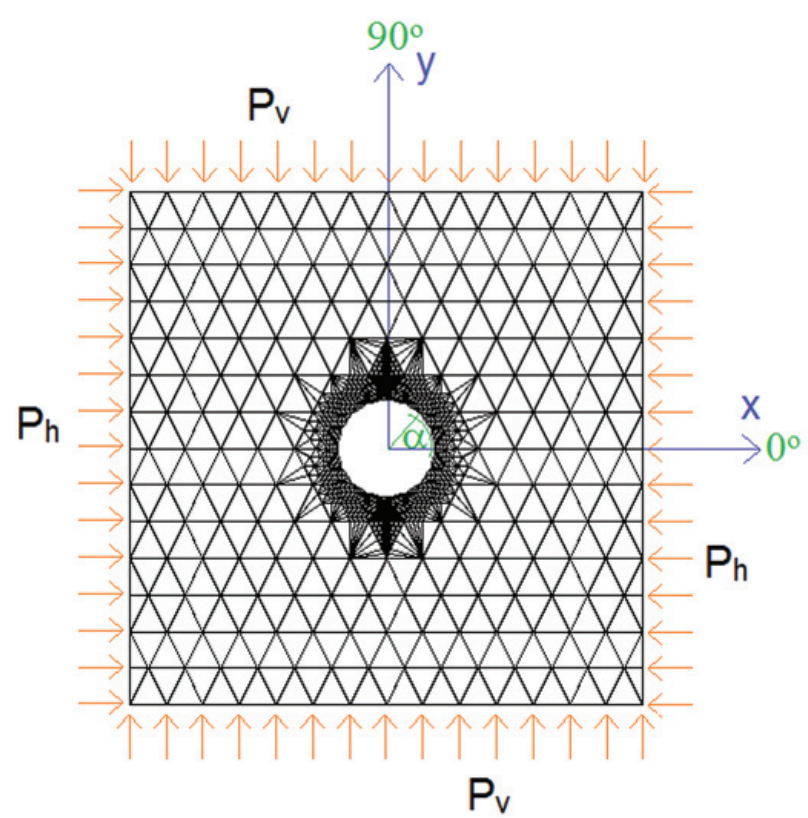

Figura 19. Remalleo final, malla de 1692 elementos y 908 nudos

Comparación de resultados. En las figuras 20 a 22 se presentan las gráficas de los desplazamientos en la circunferencia del orificio obtenidos mediante elemento finito para las distintas mallas y la solución analítica.

De las gráficas de desplazamientos puede verse que el tamaño de los elementos influye en la aproximación a la solución analítica; con el remalleo fractal se tiene

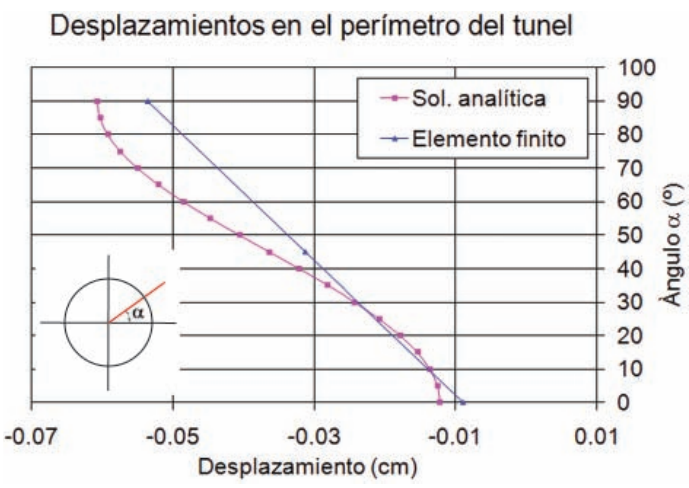

Figura 20. Desplazamientos en la circunferencia, malla burda 


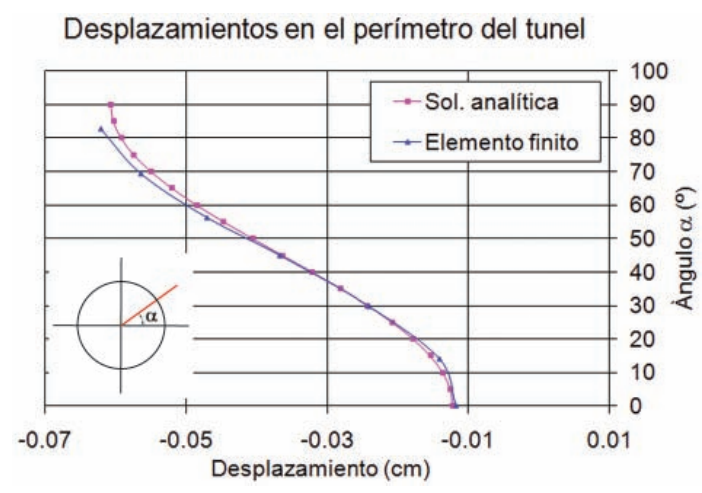

Figura 21. Desplazamientos en la circunferencia, malla fina

\section{Desplazamientos en el perímetro del tunel}

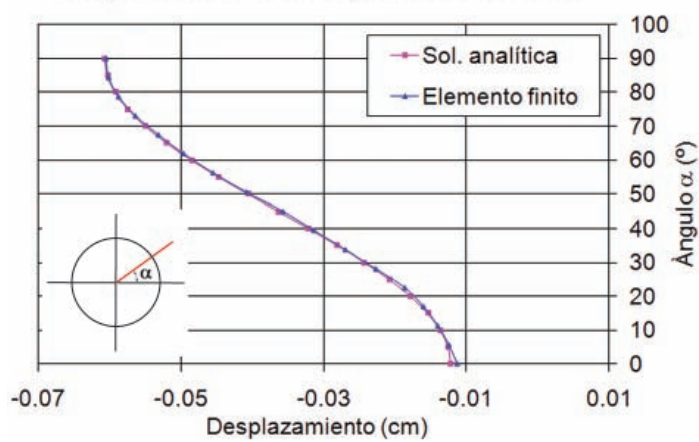

Figura 22. Desplazamientos en la circunferencia, remalleo fractal

una excelente aproximación a la solución analítica debido al grado de refinamiento automático de la malla en las zonas cercanas a la pared del túnel.

También resulta evidente que en el remalleo se obtiene mejor aproximación que en la malla fina. Esto indica que el remalleo, al ser automático refina la malla lo necesario para lograr resultados idénticos a los de la solución analítica.

\section{Solución analítica elasto-plástica para un túnel circu-} lar. Tresca propuso un criterio de fluencia plástica en función del esfuerzo cortante máximo (Obert y Duval, 1967).

El criterio se expresa en términos de esfuerzos principales, de tal forma que, si $\sigma_{1}>\sigma_{2}>\sigma_{3}$, el máximo esfuerzo cortante es $\left(\sigma_{1}-\sigma_{3}\right) / 2$; entonces el criterio de fluencia plástica puede determinarse de la siguiente forma:

$\frac{\sigma_{1}-\sigma_{3}}{2}=k$

donde $k$ es una constante. Esta suposición se basa en el modelo elasto-plástico perfecto de St. Venat.

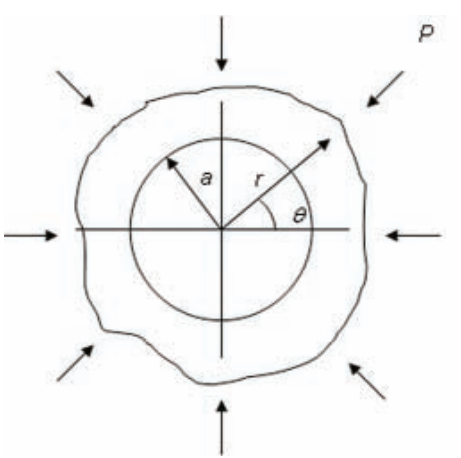

Figura 23. Túnel circular en una masa infinita bajo presión hidrostática

Análisis elasto-plástico por el método del elemento finito. Se analizó un túnel sin recubrimiento en una masa de suelo mediante el método del elemento finito. El material empleado es homogéneo y se aplicó presión radial uniforme mediante fuerzas nodales. Se despreció el peso propio. El túnel se modeló con tres mallas de elementos finitos de un sector de cuarto de círculo, teniéndose una malla burda, intermedia y fina (figuras 24 y 25). El tamaño de los elementos en cada malla aumenta radialmente, siendo más densa la malla cerca del túnel. Estas mallas, a diferencia de las utilizadas en el análisis elástico-lineal, son circulares para lograr aplicar una presión uniforme radial, como lo requiere la solución analítica, además de lograr una mayor precisión en los resultados por el método del elemento finito.

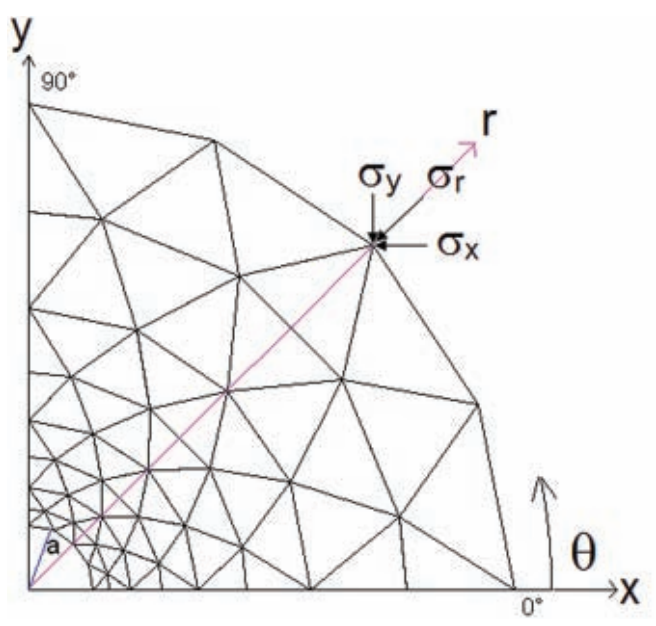

Figura 24. Malla burda de 72 elementos y 49 nodos

Después se utilizó la malla burda para aplicar el algoritmo de remalleo fractal. En la malla resultante (figura 26) puede observarse la optimización de la densidad de los elementos finitos que la conforman, pues el rema- 
lleo se concentró cerca del túnel, en donde se requiere mayor número de elementos de un menor tamaño.

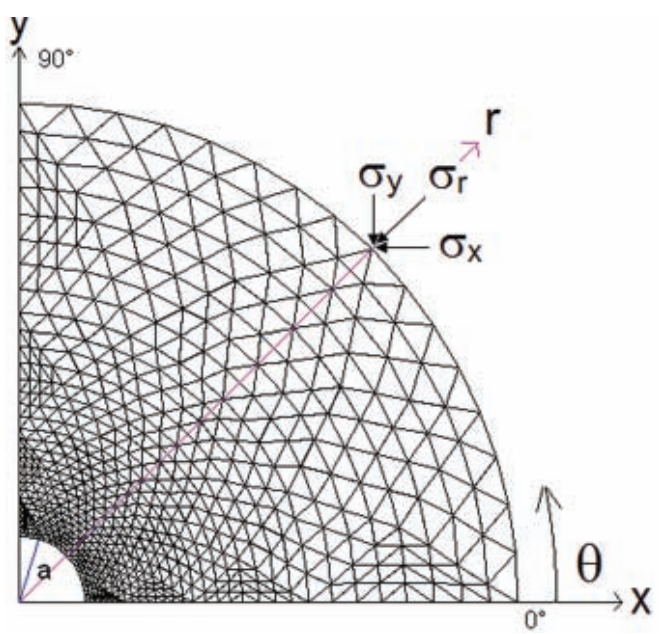

Figura 25. Malla fina de 1152 elementos y 625 nodos

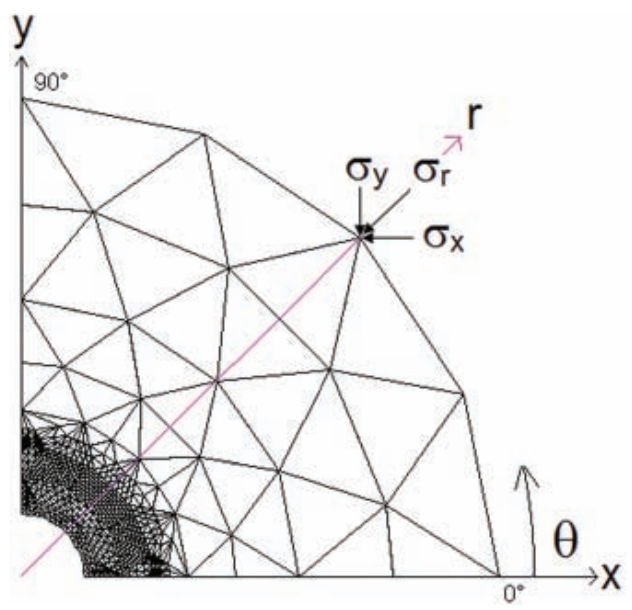

Figura 26. Malla refinada con remalleo fractal automático, 1970 elementos y 1036 nodos

Comparación de resultados obtenidos. A continuación se presentan las graficas de comparación entre los resultados obtenidos con la solución analítica y el método del elemento finito para las mallas presentadas anteriormente (figuras 27-29). Los resultados corresponden a los esfuerzos verticales $\sigma_{y}$ (proyectados de $\sigma_{r}$ ) sobre una línea a $45^{\circ}$.

En la figura 29 se observa que los resultados obtenidos por el método del elemento finito (con remalleo) se aproximan bastante a la solución analítica y el quiebre en el límite entre la zona plástica y la elástica está bien definido. Los resultados cerca del túnel son más aproximados a la solución analítica, viéndose claramente el

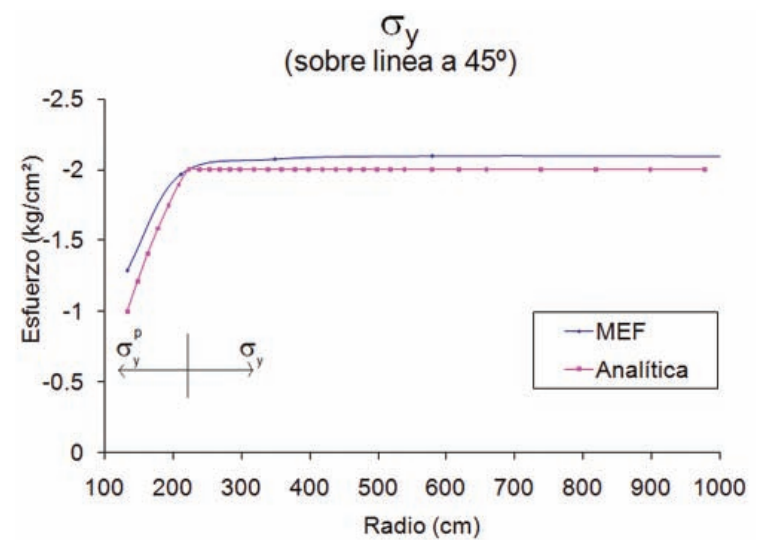

Figura 27. $\sigma_{y}$ (línea a 45oㅡ), malla burda

efecto de la densidad de los elementos en la periferia del túnel. Sin embargo, en zonas alejadas, la malla es mucho más burda (figura 26), por lo que los resultados lejos del túnel son ligeramente menos aproximados.

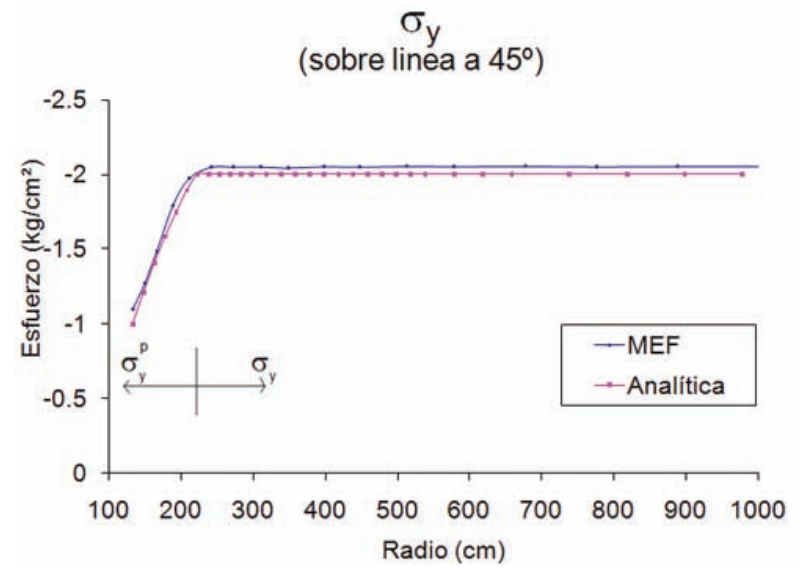

Figura 28. $\sigma_{y}$ (línea a 45으), malla fina

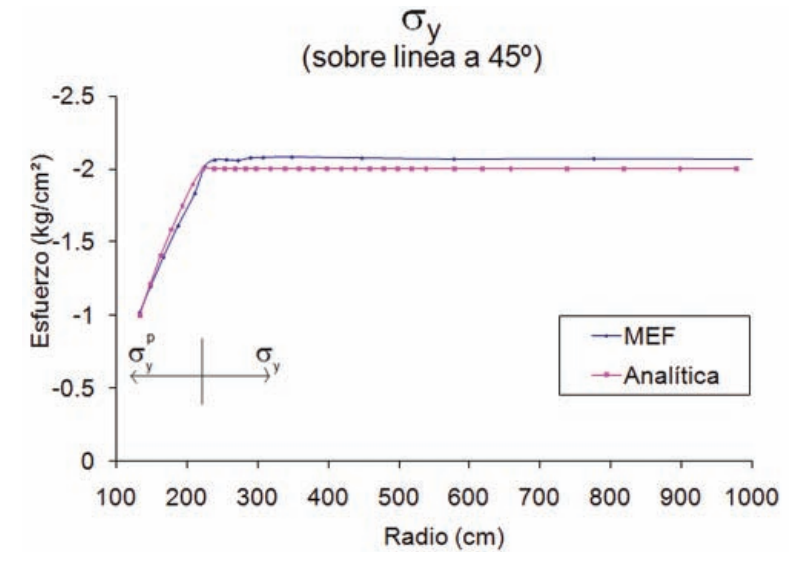

Figura 29. $\sigma_{y}$ (línea a 45oㅡ), malla remallada 
Esto se cambiaría simplemente definiendo un criterio más severo para iniciar el remalleo en la zona externa.

Solución analítica elástico-lineal para una carga vertical uniforme sobre un espacio semi-infinito

La solución analítica al problema de una carga vertical de ancho finito y longitud infinita sobre un espacio semi-infinito (figura 30) está dada por las ecuaciones:

$\sigma_{z}=\frac{q}{\pi}(\beta+\operatorname{sen} \beta \cos (\beta+2 \delta))$

$\sigma_{x}=\frac{q}{\pi}(\beta-\operatorname{sen} \beta \cos (\beta+2 \delta))$

donde:

$q$ : $\quad$ carga por unidad de longitud

$\delta, \beta: \quad$ ángulos

$\sigma_{z}, \sigma_{x}: \quad$ esfuerzos normales

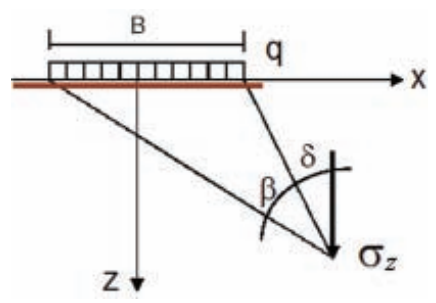

Figura 30. Esfuerzo $\sigma_{z}$ debido a una carga vertical uniforme

Análisis elástico-lineal por el método del elemento finito. Se modeló el problema de una carga uniforme vertical actuando sobre una masa de suelo mediante una malla de elementos finitos. Dicha malla se sometió al proceso de remalleo fractal (cuatro iteraciones), obteniéndose las configuraciones mostradas en las figuras 31 y 32.

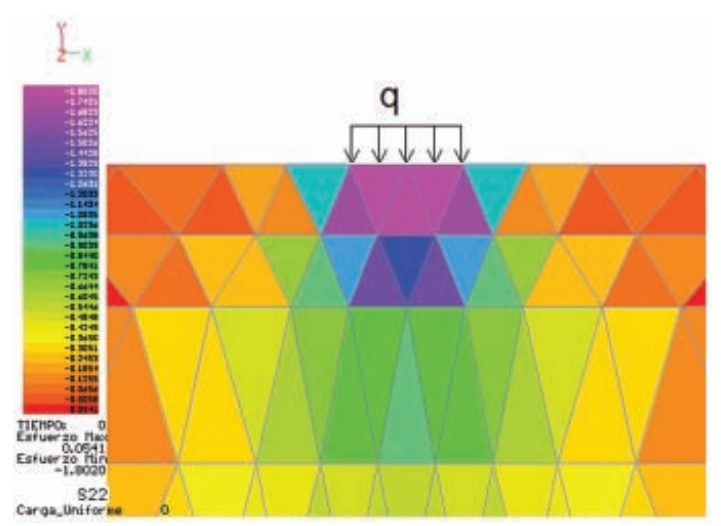

Figura 31. Esfuerzo $\sigma_{z}$ en la malla inicial

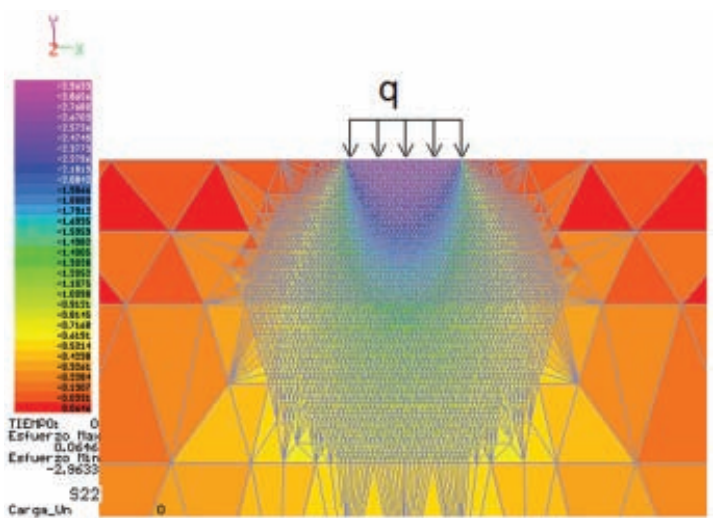

Figura 32. Esfuerzo $\sigma_{z}$ en el cuarto remalleo

Comparación de resultados obtenidos. A continuación se presentan las gráficas de comparación entre los resultados obtenidos con la solución analítica y el método del elemento finito para la malla presentada antes sujeta a remalleo. Las curvas muestran los esfuerzos verticales actuando a lo largo de la sección media del suelo de cimentación.

En las figuras 33 y 34 puede observarse cómo, mediante el remalleo fractal, la solución numérica se

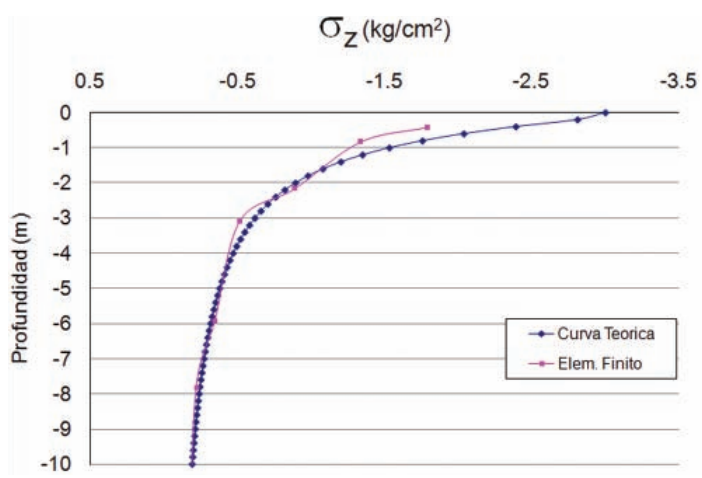

Figura 33. Esfuerzos verticales, malla inicial

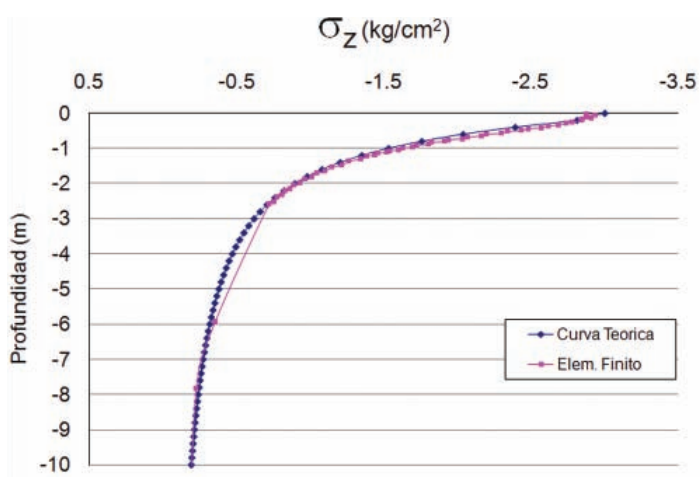

Figura 34. Esfuerzos verticales, 4o remalleo 
aproxima a la solución teórica, muy cerca de la zona de aplicación de carga (contacto suelo-cimentación), pues es donde la concentración de esfuerzos es mayor.

\section{Conclusiones}

El empleo de algoritmos fractales en el remalleo automático de mallas de elementos finitos permite detectar zonas críticas o inestables en el medio continuo, que bajo ciertas condiciones de esfuerzo-deformación pueden revelar la fluencia de los materiales y el posible origen de grietas. El algoritmo de remalleo fractal tiene la propiedad de que es muy simple y puede aplicarse reiteradamente de manera automática hasta obtener formas muy complejas en las mallas de elemento finito, que modelan con muy buena aproximación el comportamiento de obras reales.

Dependiendo del criterio de falla utilizado para efectuar el remalleo, la configuración de la malla remallada indicará en qué regiones se presenta la fluencia de los materiales. Estos criterios son muy variados y cada uno de ellos influirá en forma muy distinta en el remalleo.

Las gráficas mostradas en los análisis elástico-lineal y elasto-plástico realizados al túnel revelan que la aproximación de los resultados depende en gran medida de la densidad de la malla de elementos finitos y el tamaño de éstos; con el remalleo se comprobó que es necesario afinar aquellos sitios en donde los gradientes de esfuerzos son grandes, o bien en donde los esfuerzos normales y cortantes son máximos, pues es ahí en donde se requiere más información para analizar aproximadamente el problema con elementos finitos.

El remalleo también depende del tipo de análisis que se realice, ya que los análisis elasto-plásticos y lineal muestran formas distintas; esto se debe a que en cada tipo de análisis las propiedades mecánicas de los materiales se modifican de acuerdo a diferentes leyes de esfuerzo-deformación. Esto implica una redistribución de esfuerzos y deformaciones distintas conforme al modelo de comportamiento del suelo.

\section{APÉNDICE}

Campos de las matemáticas modernas vinculados con los fractales.

En este apéndice se mencionan algunos campos de las matemáticas de los cuales es recomendable manejar algunos conceptos básicos para comprender mejor la naturaleza de los fractales.

\section{Conjuntos}

La importancia de la teoría de conjuntos radica en que se pueden definir los siguientes conceptos y probar todas sus propiedades: par ordenado, relación, función, partición, orden, estructuras algebraicas, los naturales, los enteros, los racionales, los reales, los complejos, etcétera.

En general el concepto de conjunto obliga a reflexionar en dos cuestiones íntimamente vinculadas, por un lado en una propiedad determinada de un objeto y en segundo lugar en la formación de un conjunto a partir de todos los objetos que poseen esa característica.

\section{Topología}

La topología es una de las ramas matemáticas que a su vez se utiliza en muchas otras ramas de las matemáticas, permitiendo entre todas ellas una conexión coherente. Se puede considerar que una transformación topológica es semejante al concepto físico de deformación, lo cual no es exactamente correcto. Entre las definiciones importantes, se tienen: la de vecindad, cercanía, conjunto denso, o no denso, conjunto cerrado o abierto si incluye o no su frontera, etcétera.

Lo relevante en este caso es que la topología estudia aquellas transformaciones en las cuales las imágenes de los objetos no sufren cambios que alteren su estructura topológica, es decir, por ejemplo, un cuerpo con un orificio como una dona, topológicamente es análogo a una taza, que también tiene un orificio en el asa de donde ésta se sujeta.

\section{Estructuras algebraicas}

Una estructura algebraica es un conjunto con una o varias operaciones, que satisfacen unos axiomas dados. Cualquier operación por elemental que sea implica un mapeo (o transformación matemática). Entre las estructuras algebraicas más usadas se tienen: magmas, semigrupos, grupos, anillos, etcétera.

En este concepto lo importante es comprender que existe una gran variedad de opciones para realizar operaciones diversas, con elementos de conjuntos muy generales, y no simplemente pensar en las operaciones del algebra clásica, como suma, resta, multiplicación.

\section{Álgebra de boole}

Se tiene que la lógica o el álgebra de Boole (y sus operadores lógicos $\mathrm{Y}, \mathrm{O}$ y NO) al ser también una estructura algebraica, implica que en los razonamientos lógicos también se definen operaciones específicas. 


\section{Álgebra sigma}

Es una colección de subconjuntos del espacio muestra (conjunto de resultados posibles de un experimento) que contiene el conjunto vacío ø y es cerrada bajo uniones contables.

Ejemplo: lanzamos un dado al aire, observamos el número de puntos en la cara superior. El espacio muestral del experimento es $S=\{1,2,3,4,5,6\}$. Uno de los álgebra sigma que podemos asociar a $S$ es la colección $\{\varnothing\},\{1,2\},\{3,4,5,6\}$, S. En síntesis, las operaciones son uniones, intersecciones y otras, semejantes a las que se emplean en teoría de probabilidades, ya que ésta pertenece a este tipo de álgebras.

Aquí se debe resaltar que estas álgebras, son conjuntos en los que sus elementos se forman a partir de una serie de objetos elementales, combinándolos, de manera semejante a un número de dos o más dígitos a partir de una base dada (binaria, decimal, hexadecimal, etcétera) o también cómo se forman las palabras a partir de las letras del alfabeto.

\section{Álgebra de borel}

Finalmente un álgebra de Borel se considera parte de las estructuras algebraicas e implica una iteración de mapeos. Es una de las dos álgebras-sigma siguientes:

- La mínima álgebra-sigma que contiene los conjuntos abiertos.

- La mínima álgebra-sigma que contiene los conjuntos cerrados.

En este caso los elementos base tienen características geométricas, por ejemplo, segmentos de línea, círculos, entre otras, y mediante ellos se hacen combinaciones. Por ejemplo, cómo se combinan para formar letras (A, B, C, D, etcétera.), así la A es combinación de tres segmentos rectilíneos, la $\mathrm{D}$ de un segmento lineal y de un arco circular, y así las demás letras. Por este motivo el manejo de fractales sigue reglas semejantes a este tipo de álgebra.

\section{Álgebra operacional}

Aquí se generaliza la idea de operación al grado de operadores que actúan incluso sobre otros operadores. Por ejemplo, se tienen las siguientes propiedades de estas operaciones.

Propiedad de multiplicación. El producto de los operadores de dos funciones entrantes $f()$ y $g()$ es igual al producto del operador D con el operador de la función $f^{*} \mathrm{~g}()$ :

$[f][g]=D\left[f^{*} g\right]$

Propiedad aditiva. Un operador $\mathrm{V}$ se dice que es aditivo si cumple la igualdad

$V\left[\varphi_{1}+\varphi_{2}\right]=V \varphi_{1}()+V \varphi_{2}$

Basándose en esta álgebra, se pueden construir "operaciones" muy generales, no únicamente de tipo numérico.

Teoría de la medida

Entre los objetivos de este inciso se encuentran: conocer las propiedades de las medidas (en general), así como de la integral de Lebesgue. También, manejar los conceptos básicos de espacios abstractos de medida (empleados, por ejemplo, en teoría de probabilidad).

En matemáticas una medida es una función (o mapeo u operador) que asigna un número, por ejemplo, a un "volumen" o "probabilidad", a subconjuntos de un conjunto dado (es decir, son funciones que implican manejos algebraicos diferentes según el conjunto al que se aplican).

La característica básica de esta teoría es poder definir una regla operativa tal sobre las propiedades de los objetos de un conjunto dado, como resultado de una imagen que es simplemente una escalar.

Medida e integración. La teoría de medida e integración es el estudio de longitudes, superficies y volúmenes en espacios generales.

La integral de Lebesgue. Este tipo de integral cubre una clase más amplia de funciones que la integral de Riemann. Esta integral equivale a una extensión de las nociones clásicas de longitud y área a conjuntos más complicados. A partir de conceptos similares se construye la medida de Hausdorff, que es básica en la caracterización de los conjuntos fractales. Debe recalcarse que las diferentes integraciones posibles son casos particulares dentro de la teoría de la medida.

\section{Geometría algebraica}

La geometría algebraica estudia los sistemas de ecuaciones polinómicas con coeficientes. Observe que se relega a un segundo plano los sistemas de ecuaciones para centrarse en las estructuras algebraicas derivadas de sus soluciones, es decir, consiste en centrar la aten- 
ción en los conjuntos de soluciones de los sistemas, los cuales forman conceptos matemáticos, interpretables geométricamente como puntos, rectas, planos y generalizaciones a dimensiones superiores.

La característica esencial de esta geometría (que es un álgebra no lineal) es que las soluciones en este caso son múltiples y no únicas como en el álgebra lineal. Esto da origen a bifurcaciones, etcétera.

\section{Referencias}

Barnsley M.F. Fractals Everywhere. Academic Press. New York. 1990.

Basualdo Cátedras de geotecnia y cimentaciones 2005. [en línea]. Disponible en: http://www.carreras.frba.utn.edu.ar/civil/geotecnia/Analisis\%20no\%20lineal\%20Rev.1.pdf.

Falconer K. Fractal Geometry. Mathematical Foundations and Applications. John Wiley and Sons, Chichester. 1993.

Gutiérrez J. Sistemas no lineales. Conceptos, algoritmos y aplicaciones. V Conferencia Nacional de Ciencias de la Computación, CCBOL'98. Potosí, Bolivia, noviembre, 1998.
Guzmán M., De Martín M.A., Moran M., Reyes M. Estructuras fractales y sus aplicaciones. Editorial Labor. Barcelona. 1993.

Hermosillo A. Refinamiento automático de mallas de elemento finito mediante teoría de fractales y su aplicación en problemas geotécnicos (tesis de maestría, DEPFI, UNAM), 2006.

Magaña R., Pérez M., Hermosillo A., Romo M., Comparative Studies Between Methods of Classic Remeshing and Fractal Approaches, International Conference on Adaptaptative Modeling and Simulation, ADMOS 2003, Göteborg, Suecia, septiembre 2003.

Magaña R., Pérez M. y Romo M. Uso de fractales en el refinamiento continuo de mallas de elemento finito, XXI Congreso Nacional de Ingeniería Sísmica, Guadalajara, México. 2001

Muñoz F., Meza R. Nubes fractales: varias aproximaciones. Proyecto de Computación Gráfica - CC52B. Dpto. de Cs. de la Computación. FCFM - U. de Chile. 2007.

Obert L., Duvall-Wilbur I. Rock Mechanics and the Design of Structures in Rock. Editorial John Wiley and Sons, 1967.

Solís C. Fundamentos y métodos activos para el aprendizaje de la matemática. Primera edición. Huancayo Perú Sección: Matemática publicado por: Miguel Guzmán, noviembre, 2004.

\section{Semblanza de los autores}

Roberto Magaña Del Toro. Ingeniero Civil (1970) por la Facultad de Ingeniería, UNAM. M. I. (geotecnia) por la DEPFI, UNAM. Estudios de doctorado completos en la misma institución. Es Técnico Académico Titular C, en el Instituto de Ingeniería, desde hace 35 años. Ha escrito 62 artículos para congresos nacionales e internacionales. Ha impartido materias y seminarios en la Facultad de Ingeniería y en la DEPFI de la UNAM. Línea de investigación: aplicación de métodos numéricos y elemento finito al estudio del comportamiento dinámico de obras geotécnicas. Nuevas líneas de investigación: aplicación de geometría fractal para el remalleo automático de mallas de elemento finito, uso de la teoría del caos y de las ecuaciones diferenciales fraccionarias para el estudio de señales sísmicas.

Armando Rafael Hermosillo Arteaga. Ingeniero Civil por la Facultad de Ingeniería de la UNAM en 2004. Maestro en Ingeniería por la misma Facultad en 2006. Es becario del Instituto de Ingeniería de la UNAM desde septiembre de 2001. La línea de investigación en la que colabora se relaciona con la aplicación de métodos numéricos, método de elementos finitos, teoría del caos y fractales en la solución de problemas de ingeniería geotécnica. Ha participado como coautor en la elaboración de 15 artículos para congresos nacionales e internacionales. Actualmente se encuentra realizando sus estudios de Doctorado en la Facultad de Ingeniería, UNAM.
Miguel Pedro Romo Organista. Ingeniero Civil por la Universidad Autónoma de Guadalajara (1968). Maestría en Mecánica de Suelos por la UNAM en 1972 y Doctorado en Ingeniería Geotécnica por la Universidad de California, Berkeley en 1976. Profesor Investigador Titular del Instituto de Ingeniería, UNAM desde 1977. Premio Universidad Nacional 2005 en el área de Innovación Tecnológica y Diseño Industrial, UNAM, Premio "Nabor Carrillo Flores 2004-2005" del Colegio de Ingenieros Civiles de México y Premio Nacional de Ciencias y Artes 2007. Ha dirigido 10 tesis de doctorado, 32 de maestría y 9 de licenciatura. Investigador Emérito del Sistema Nacional de Investigadores (SNI).

Jorge Carrera-Bolaños. Licenciado en matemáticas por la Universidad de Leipzig, Alemania. Doctor en matemáticas aplicadas (teoría de sistemas) por la universidad L. Eotovos, Budapest, Hungría. Doctor en filosofía de la ciencia, UNAM. Profesor Titular C, TC, Facultad de Ingeniería, UNAM. Más de 15 publicaciones internacionales, la mayoría arbitradas. Ha participado con ponencias en más de 80 congresos nacionales e internacionales. Tutor de 5 tesis de doctorado y diversas de maestría y licenciatura. 